\title{
DIALOGIA MÃE-FILHO EM CONTEXTOS DE DEPRESSÃO MATERNA: REVISÃO DE LITERATURA
}

\author{
Mother-Child Dialogism in Maternal Depression Context: \\ Literature Review
}

Janaína Pereira Pretto Carlesso (1), Ana Paula Ramos de Souza (2)

\begin{abstract}
RESUMO
Tema: o presente estudo analisou a produção científica dos últimos cinco anos sobre a depressão materna, em particular as repercussões da depressão na interação mãe-filho e as suas implicações para o desenvolvimento infantil. Objetivo: verificar as repercussões da depressão materna na interação mãe-filho, e examinar suas implicações para o desenvolvimento da criança, especialmente o de linguagem. Conclusão: a revisão de literatura realizada demonstra que a depressão materna afeta não só a mãe, mas também o desenvolvimento global do bebê. Os estudos apontaram que os efeitos da depressão materna podem resultar negativamente no desenvolvimento da criança, potencializando desordens lingüísticas, comportamentais, afetivas, cognitivas e sociais. Portanto, a depressão materna, em um determinado momento após o nascimento, pode interferir no estabelecimento do vínculo mãe-bebê e, por isso, deve ser tratada o mais precocemente possível.
\end{abstract}

DESCRITORES: Depressão Pós-Parto; Desenvolvimento Infantil

\section{INTRODUÇÃO}

A depressão materna pós-parto é um dos aspectos importantes a serem considerados como possível interveniente no exercício da função materna durante o primeiro ano de vida do bebê. Ela pode ter conseqüências importantes no desenvolvimento infantil, sobretudo de linguagem cuja estruturação se dá pela interação dialógica mãefilho ${ }^{1}$.

O processo comunicativo precoce da mãe-bebê tem papel decisivo na formação vincular da díade, pois é um intercâmbio não somente de informações que guiam à aquisição da linguagem verbal, mas também dos sinais afetivos. A interação natural e espontânea com a figura materna é fundamental

(1) Psicóloga; Especialista em Educação Especial: Altas Habilidades Superdotação pela Universidade Federal de Santa Maria, UFSM, Santa Maria, RS; Mestranda em Distúrbios da Comunicação Humana UFSM - Santa Maria- RS, Brasil.

(2) Fonoaudióloga; Docente do Departamento de Fonoaudiologia da Universidade Federal de Santa Maria, UFSM, Santa Maria, RS; Doutora em Letras pela Pontifícia Universidade Católica do Rio Grande do Sul.

Conflito de interesses: inexistente neste processo, pois através da fala, do toque e os cuidados corporais, a mãe conduz o bebê a emitir progressivamente suas primeiras expressões comunicativas no diálogo ${ }^{2}$.

No entanto, esta naturalidade parece ficar abalada quando a criança demora a falar. Por isso, quando algum fator interfere na aquisição da linguagem durante os primeiros anos é necessário que haja uma intervenção o mais cedo possível, visto que o período de plasticidade cerebral maior se dá nesse período. Não são todas as condições que afetam a evolução da linguagem, tendo efeitos permanentes e vitalícios, mas certas condições podem durar o suficiente para impedir que certos estágios do desenvolvimento se manifestem. Portanto, eventos adversos nos primeiros anos de vida podem provocar a regressão ou a paralisação do desenvolvimento da linguagem ${ }^{3}$. Indivíduos que sofreram privações extremas na primeira infância como maus tratos e negligência podem apresentar carência de atributos humanos básicos como a fala e habilidades sociais ${ }^{4}$.

Recchia e Ramos ${ }^{5}$ apresentaram alguns casos de retardo de aquisição da linguagem, em que havia indícios de depressão materna no período do 
pós-parto. Havia alterações do vínculo mãe-filho, que se refletia na quase ausência de respostas da mãe nos diálogos da díade. Em outros havia uma descolagem incompleta da mãe-filho, pelo não comparecimento da função paterna para impor o corte necessário. Em todos os casos, ficaram claras as relações entre as alterações na dialogia mãe-filho e alterações no exercício das funções parentais. Tais alterações parecem estar relacionadas às observações de Frizzo e Piccinini ${ }^{6}$ de que a depressão materna, no período pós-parto, pode afetar a contingência da interação mãe-bebê. Os autores demonstraram que até mesmo as formas mais brandas de depressão da mãe podem afetar o bebê, na medida em que ele perceberia as mínimas deficiências da interação com sua mãe. Com base na literatura, grande parte dos estudos sobre a interação bebê-mãe deprimida abordam as implicações da depressão pós-parto na interação nos primeiros meses após o nascimento da criança, considerando-se a maior incidência dos quadros depressivos nesse período ${ }^{7}$. Considerando os pressupostos preliminares sobre a relação mãebebê como aspecto fundamental no desenvolvimento infantil, sobretudo nos aspectos linguísticocognitivos, ressalta-se a importância do estudo das repercussões da depressão materna na dialogia mãe-bebê. Por isso, este estudo tem o objetivo de verificar as repercussões da depressão materna na interação mãe-filho, e examinar suas implicações para o desenvolvimento da criança, especialmente o de linguagem, em estudos recentes sobre o tema, relatados em periódicos indexados em bases nacionais e internacionais.

\section{METÓDO}

Realizou-se uma revisão de literatura sobre as repercussões da depressão materna na dialogia mãe-filho e as suas implicações para o desenvolvimento infantil. Para isso, procedeu-se uma busca sistemática das principais produções sobre a temática nos últimos cinco anos, nas bases de dados do Medline, Lilacs e Scielo, por meio da palavrachave "depressão materna". Foram identificados 30 artigos científicos no Scielo, 10 no Medline e 81 no Lilacs. Após este procedimento, realizou-se a leitura de todos os artigos identificados. Como critérios de inclusão nesta revisão, utilizou-se a busca de artigos que considerassem os efeitos da depressão materna na constituição psíquica e da linguagem do bebê. Para a análise dos dados foram considerados os principais achados e seu significado para o desenvolvimento infantil. Deste total de 121 artigos encontrados foram selecionados 22 artigos científicos. Para o relato da análise foram selecionadas duas categorias que agrupavam os artigos em temáticas: função materna e depressão puerperal, e repercussões da depressão materna no bebê.

\section{REVISÃO DE LITERATURA}

\section{Função Materna e Depressão Puerperal}

A depressão materna no pós-parto tem consequências importantes para a criança e em diversas áreas do desenvolvimento, afetando a formação do vínculo na díade mãe-bebê ${ }^{8}$, o desenvolvimento neurológico, cognitivo e psicológico na infância ${ }^{9} \mathrm{e} o$ desenvolvimento sócio-emocional na adolescência 10. O aumento de transtornos emocionais na infância de crianças cujas mães apresentaram depressão grave é significativo, sendo necessárias estratégias de prevenção dirigidas a estes grupos ${ }^{11}$.

Estudos sobre os transtornos emocionais no pós-parto identificam diversas características do comportamento e do estado emocional da mãe na fase puerperal, como transtorno de estresse póstraumático, depressão pós-parto e psicoses puerperais $^{12}$. A presença de conflitos emocionais está associada a fatores fisiológicos e emocionais e a situações de vida da mulher, como dificuldades do casal, pouco suporte familiar ou não querer engravidar $^{13}$.

Dentre os eventuais transtornos emocionais do pós-parto, três podem ser enfatizados: melancolia da maternidade (baby blues), psicose puerperal e depressão. Algumas mulheres, em torno do terceiro dia após o parto, apresentam depressão precoce ou baby blues, um estado de fragilidade e hiperemotividade (disposição a reagir emocionalmente de modo excessivo às ocorrências). $\mathrm{O}$ choro e a tristeza são acompanhados por sentimentos de falta de confiança e incapacidade para cuidar do bebê. O baby blues corresponde a uma etapa de reconhecimento mútuo entre a mãe e o bebê. É o tempo necessário para a mãe compreender que o bebê é um ser separado dela, marcando o fim da gravidez psíquica ${ }^{14}$.

A depressão pós-parto ocorre após o baby blues, sendo que os sentimentos depressivos não cedem ao final de duas semanas após o parto. É uma manifestação psicopatológica importante, pois cerca de 10 a $15 \%$ das mulheres apresentam um quadro depressivo dentro dos três primeiros meses após o parto. Geralmente, manifestam-se sentimentos de incapacidade de cuidar do filho e dificuldades para enfrentar a nova configuração sóciofamiliar. A sintomatologia típica inclui: sentimentos de culpa, transtornos do sono, flutuações de humor com grande tendência a tristeza e ausência de sintomas psicóticos ${ }^{14}$. 
Em termos de entendimento psicodinâmico, o nascimento da criança representa o rompimento da relação simbiótica entre o bebê e a mãe. Este processo de separação pode desencadear na mãe vivências depressivas e psicóticas, reativadas por conflitos e lutos mal-elaborados da infância. Nesse momento a mãe precisa desenvolver um vínculo afetivo, que the permitirá identificar-se com a criança, colocando-se no lugar dela e imaginando o que ela está necessitando ${ }^{15}$. Para Alt e Silvia ${ }^{16}$, o parto envolve a separação de dois organismos que estavam vivendo juntos em uma relação de total dependência e permanente contato íntimo. Ao nascer, a criança encarrega-se de uma variedade de funções fisiológicas que até então eram cumpridas pela mãe, como a respiração, a alimentação e outras. A mãe, que se adaptara ao estado de gravidez e incorporara o feto no seu esquema corporal, deverá passar por um novo processo de ajustamento, retornando a situação de nãogravidez. A angústia no momento do parto é de natureza catastrófica, pois há, na mulher, um sentimento de perda e de esvaziamento de partes importantes de si mesma e medo de ser atacada por algo desconhecido (bebê). Já a etapa do puerpério é caracterizada pela dualidade entre a situação do perdido, gravidez, e do adquirido, o bebê.

Os serviços de saúde têm grande preocupação com a depressão materna, especialmente pelas consequências para o desenvolvimento infantil. As mães deprimidas apresentam, de forma mais acentuada, impressões e sentimentos negativos com relação às crianças e à maternidade do que as mães sem depressão ${ }^{17}$. No geral, o estresse vivenciado por essas mães exacerba seus níveis de ansiedade e depressão e dificulta seu ajustamento e o desempenho de seu papel de cuidadora, na medida em que a depressão tende a afetar a disponibilidade cognitiva e emocional da mãe e, consequentemente, sua responsividade à criança ${ }^{18}$.

Os efeitos da depressão materna não se limitam ao atraso no desenvolvimento dos primeiros tempos de vida, mas podem ocasionar alterações na interação mãe-filho na primeira infância e causar prejuízos no desenvolvimento cognitivo e comportamental, a longo prazo ${ }^{19}$.

\section{Repercussões da Depressão Materna no Bebê}

Muitos estudos sugerem que as manifestações comportamentais associadas à depressão materna podem ocorrer muito cedo no desenvolvimento do bebê e repercutir ao longo de sua vida. Uma revisão de artigos ${ }^{20}$ sobre os efeitos da depressão materna no desenvolvimento infantil, corrobora os dados encontrados na pesquisa de que a depressão materna apresenta efeitos a longo prazo no desenvolvimento infantil ${ }^{21}$. Os pesquisadores afirmam que crianças de mães deprimidas apresentam $29 \%$ de chance para desenvolver desordens emocionais e comportamentais quando comparadas com $8 \%$ de chance de crianças de mães não deprimidas. Igualmente, o contato com mães deprimidas, no primeiro ano de vida, é responsável pela baixa habilidade cognitiva da criança aos 4 anos de idade.

Frizzo e Piccinini ${ }^{22}$ realizaram a filmagem dos bebês de 3 a 6 meses na interação face a face com suas mães e com um estranho. Os autores avaliaram a performance comportamental dos bebês através de uma escala de medida de interação. Os bebês de mães deprimidas demonstraram uma interação mais pobre não só com suas mães, mas também quando interagiram com estranhos. Em associação à freqüência cardíaca mais elevada, os bebês de mães deprimidas apresentaram uma menor vocalização $(p<0,001)$ e mais freqüentemente desviaram o olhar $(p<0,01)$ na interação, tanto com suas mães como com estranhos em relação ao grupo controle. A maioria dos comportamentos não diferiu em ambos os grupos, exceto por apresentarem maior aversão ao olhar (olhavam ao longe) e menor orientação da cabeça em direção à mãe $(p$ $<0,001)$, sugerindo que fatores ambientais estejam envolvidos nos resultados.

Goldbort ${ }^{23}$ avaliaram a interação de mães deprimidas com seus bebês de 6 a 7 meses. Eles subdividiram o comportamento materno em três padrões: retirado, intrusivo ou adequado. A interação foi realizada através do paradigma face a face por 3 minutos e, após, durante 40 minutos na interação espontânea. Quando retiradas, as mães brincavam pouco com seus bebês, raramente falavam com eles na linguagem de bebês e tinham um afeto triste $e$ pouco modulado. As mães intrusivas expressavam irritação para com seus bebês e interferiam em suas atividades espontâneas e próprias. As mães mais adequadas eram capazes de interagir positivamente com seus bebês. Foram utilizados descritores comportamentais durante a interação face a face, codificados e analisados segundo a segundo. Os autores encontraram diferenças notáveis nos bebês conforme a mãe fosse retirada ou intrusiva. Os bebês de mães retiradas eram angustiados, irrequietos, procuravam engajamento sem sucesso e gastavam mais tempo chorando, isto é, protestavam significativamente mais. Os bebês de mães intrusivas evitavam significativamente mais o olhar (olhavam ao longe) e o interagir com suas mães. Ambos os grupos de bebês tinham uma interação significativamente menos positiva $(p<0,001)$ em relação ao grupo dos bebês de mães deprimidas socialmente positivas (adequadas). Associado a 
isso, as mães socialmente positivas apresentavam uma maior amplitude de comportamento e afeto.

Lee et al. ${ }^{24}$ encontraram, numa amostra de mães deprimidas, uma persistência da depressão em $70 \%$ delas ainda aos 6 meses de vida do bebê. Esses bebês, aos 12 meses, demonstraram um atraso no desenvolvimento (avaliado através da escala de Bayley), sinais neurológicos leves, menor comportamento exploratório e percentil de peso mais baixo.

Akman et al. ${ }^{25}$ observaram afetos menos positivos em bebês de 3 meses de mães deprimidas, sugerindo um comportamento depressivo já num período inicial de vida dos bebês. As duplas foram filmadas na interação face a face por 3 minutos. Para a avaliação das expressões faciais dos bebês, foi utilizado o sistema AFFEX (system for identifying effect expressions by holistic) (Izard \& Dougherty, 1980), no qual são codificadas 10 expressões faciais (interesse, alegria, raiva, tristeza, surpresa, medo, angústia, desagrado, auto-conforto, aversão ao olhar/olhar ao longe). Os bebês das mães deprimidas obtiveram escores significativamente mais altos de expressões negativas. Os bebês de mães deprimidas ficaram significativamente mais tempo da interação com expressões de tristeza $(p<0,01)$ e raiva $(p<0,01)$ e menos tempo demonstrando interesse $(p<0,05)$ por suas mães. Esses bebês demonstraram preferência por imagens de faces tristes, o que pode estar correlacionado com as expressões mais usuais de suas mães.

Em um grande estudo longitudinal foram acompanhadas 1.215 duplas mãe-bebê ${ }^{26}$ a partir do nascimento até os 36 meses. As mães foram avaliadas .e categorizadas (CES-D; Radloff, 1977) como tendo depressão crônica, depressão com intervalos e sem depressão. As crianças de mães com depressão crônica, na interação com suas mães, tiveram escores significantemente mais baixos na linguagem expressiva, menos cooperativos e com mais sintomas de internalização e de externalização, do que os filhos de mães sem depressão. Os pesquisadores também avaliaram a sensibilidade materna, e observaram que mães deprimidas com baixa renda foram menos sensíveis a seus filhos quando comparadas a mães deprimidas com maior renda, principalmente quando a depressão era crônica. As evidências de que as interações face-a-face entre bebês e suas mães diferem em função da depressão pós-parto e sugerem que o estado afetivo da mãe pode repercutir também nas suas vocalizações com o bebê.

Evans et al. ${ }^{27}$ avaliou as implicações da depressão pós-parto em relação a duas características da linguagem materna: tempo de expressão vocal e pausas e o uso de entonação da voz. A autora verificou que mães deprimidas apresentavam vocalizações significativamente mais baixas para responder às vocalizações de bebês de quatro meses, assim como apresentavam mais variações vocais e pausas, e eram menos aptas a utilizar a entonação exagerada que é característica da linguagem materna.

Um aspecto avaliado nesses estudos refere-se à atenção compartilhada que a díade estabelece no momento da interação. A atenção compartiIhada está relacionada ao desenvolvimento do vocabulário, um aspecto importante para o desenvolvimento da comunicação e, consequentemente, dos relacionamentos interpessoais ${ }^{28}$. Os estudos desenvolvidos nesse sentido sugeriram que mães deprimidas podem ser menos aptas a coordenar um foco de atenção com seus filhos, na medida em que se mostraram mais preocupadas e pouco atentas às suas crianças ${ }^{29,30}$. Por exemplo, em uma investigação que comparou os modelos de atenção entre díades com mães deprimidas e não-deprimidas em uma situação que permitia a utilização tanto de um foco de atenção em comum como um foco de atenção independente, concluíram que mães deprimidas prestavam menos atenção a um evento em comum com suas crianças de dezoito a trinta meses do que mães não-deprimidas, tanto em termos de seu foco de atenção como em relação às suas atividades com a criança ${ }^{31}$.

Em experimentos realizados no Centro Médico Infantil de Boston, foi solicitado às mães de bebês de três e quatro meses de idade que se apresentam para os seus filhos com uma face tranquila, não responsiva e inexpressiva. Isto seria seguido por uma drástica mudança na aparência dos bebês. No primeiro ou segundo minuto, ele sorri, agita-se e esforça-se para obter uma resposta de suas mães. Depois de muitos esforços sem sucesso, eles ficam desestimulados e, com freqüência, começam a salivar. Depois de três a quatro minutos, todo o seu corpo mergulha em desespero. Esses foram experimentos muito breves, seguidos de abraços calorosos, mas é possível observar que a depressão de uma mãe e sua falta de respostas podem prejudicar o desenvolvimento do bebê ${ }^{32}$.

A interação mãe-bebê e sua relação com o desenvolvimento posterior da criança representam uma área de grande interesse entre os pesquisadores do desenvolvimento infantil. As investigações conduzidas nesse sentido têm enfatizado tanto as contribuições maternas quanto as contribuições do próprio bebê para a qualidade da interação ${ }^{32-34}$. Este fato pode ser verificado em métodos de observação comportamental utilizados em alguns estudos que avaliaram o impacto da depressão materna na interação com bebê em períodos mais avançados 
do desenvolvimento da criança em situações I údicas ${ }^{35}$.Alguns desses estudos examinaram os comportamentos maternos e infantis durante a exploração de brinquedos pelos bebês em situações de jogo com suas mães. De acordo com alguns estudos ${ }^{36,37}$, essa exploração por parte do bebê durante a interação com a mãe estaria associada ao seu desenvolvimento cognitivo posterior. Os comportamentos maternos de introduzir brinquedos e manter a atenção do bebê em um brinquedo pelo qual demonstrou interesse estariam associados a um aumento na exploração de objetos por parte do bebê, enquanto que o redirecionamento de sua atenção e a manifestação de intrusividade por parte da mãe estariam relacionados a um decréscimo nessa exploração. Por isso, as crianças de mães deprimidas apresentam maior risco para terem desordens comportamentais, afetivas, cognitivas e sociais, auto-imagem negativa, distúrbios do apego, maior incidência de diagnóstico psiquiátrico e de afeto negativo, bem como maior risco para apresentarem alterações da atividade cerebral ${ }^{38}$.

Mães deprimidas tendem a apresentar menos capacidade de interagir com a criança de forma adaptativa, menos capacidade de responder de forma contingente e direta aos sinais da criança e estabelecem menos contato físico com sua criança quando comparadas com mães não deprimidas. A diminuição da sensitividade materna está diretamente relacionada com o comportamento materno na interação com seu bebê que, na maioria das vezes, caracteriza-se por comportamentos de intrusividade ou de retraimento ${ }^{38}$.

No estudo realizado por Hoffman e Drotar (1991), ${ }^{39}$ sugere-se que a mãe deprimida após o parto, necessariamente não deprime o bebê. Onze mães deprimidas e onze mães não-deprimidas (entre vinte e quatro e trinta e sete anos) e seus bebês de 2 meses foram filmados em sessões de dez minutos, em que podiam interagir livremente. As mães deprimidas tiveram escores significativamente mais baixos em interação positiva, envolvimento afetivo e sensibilidade de reação, em comparação com o grupo de controle. Contrariando as hipóteses iniciais do estudo, o nível de atividade dos bebês não variou em função da depressão materna. No entanto, os bebês das mães deprimidas evidenciaram, como elas, menor grau de afeto positivo e de expressão emocional, como se identificassem com o estilo de interação das mães. Esses resultados sugerem que graus leves ou moderados de depressão materna têm efeitos seletivos na interação materno-filial. Por sua vez, graus mais intensos de depressão pós-parto podem ter efeitos a longo prazo no desenvolvimento do bebê. Este estudo mostra que esses bebês tendem a desenvolver um vínculo inseguro com as mães e a apresentar distúrbios de comportamento (especialmente no sono) até cerca de um ano e meio, mesmo quando as mães superaram a depressão em torno de três meses após o parto.

Conforme os resultados dos estudos examinados nesta revisão de literatura, pode-se observar que a mãe deprimida freqüentemente tem um padrão de cuidado do bebê não suficientemente bom, principalmente quando a depressão se estende por um período maior de tempo. A qualidade das interações parece ter sido influenciada de forma significativa pelo grau de gravidade, pela duração dos sintomas depressivos e pelo padrão comportamental da mãe com o bebê.

Embora mecanismos de ordem genética possam ter um papel nessa associação, torna-se claro que se deve considerar a qualidade da interação mãecriança e a qualidade da maternagem como contribuintes importantes para o padrão de desenvolvimento neurológico, neuroendócrino e psicológico da criança. Os estudos analisados demonstram a dificuldade da mãe deprimida em promover estímulos adequados e adaptativos que favoreçam o desenvolvimento afetivo e a aquisição da linguagem do filho. Do ponto de vista cognitivo, pode-se levar a alterações de memória e aprendizado. Além disso, podem ocorrer alterações do padrão de resposta emocional a eventos estressantes ${ }^{39,40}$.

Crestani et al. ${ }^{40}$, sugerem que a falha persistente na manipulação dos bebês nos primeiros meses de vida, principalmente a falha de um olhar responsivo da mãe e de um falar com o bebê, contribui para diminuir e/ou não desenvolver adequadamente conexões neuronais. Há evidências de que falhas no cuidado inicial devido à negligência, abuso físico e/ou psicológico estão associadas a alterações no padrão de apego e no desenvolvimento motor e mental dos bebês.

As atitudes inconscientes da mãe facilitam as ações do bebê e podem também ter uma influência patogênica sobre o seu desenvolvimento, pois "a própria perfeição de uma relação entre dois seres tão intimamente harmônicos entre si - e unidos por tantas coisas tangíveis e intangíveis - acarreta a possibilidade de sérios distúrbios, caso haja uma quebra de sintonia" ${ }^{40}$, ainda mais que essa relação envolve de um lado um parceiro ativo e dominante e do outro lado, um receptor passivo e totalmente dependente.

Conforme vários estudos 20-27,34,36,37, a depressão pós-parto pode ter sérias implicações para o bebê. Uma série de estudos mostram uma associação entre depressão materna e problemas posteriores do desenvolvimento, incluindo transtornos de conduta, comprometimento da saúde física, ligações 
inseguras e sintomas de depressão. Os sintomas de depressão pós-parto - irritabilidade, ansiedade, pouca concentração e humor depressivo - interferem em todas as relações interpessoais, especialmente no relacionamento em formação entre uma mãe e seu novo bebê. De qualquer modo não é via de regra que bebês de mães deprimidas sejam deprimidos, porém têm de duas a cinco vezes maior possibilidade de desenvolver problemas emocionais e de comportamento. Esse fato se relaciona ao fato de as mães intrusivas poderem apresentar distintos comportamentos que podem ser não-adequados, retraídos e intrusivos ${ }^{27}$.

Comportamentos maternos retraídos ou intrusivos podem gerar alterações na interação mãe-filho e tornar os bebês menos responsivos, com menores capacidades para brincar, menor vocalização ${ }^{22} \mathrm{e}$ com maior número de expressões faciais negativas $^{25}$. Como conseqüência sua prontidão escolar e seu desenvolvimento de linguagem pode estar afetados ${ }^{26}$. Essas alterações são especialmente importantes para a reflexão do fonoaudiólogo, visto que a Fonoaudiologia trabalha com bebês na triagem auditiva neonatal e pode estar observando tais comportamentos precocemente. Além disso, é o primeiro profissional a receber encaminhamento em casos de retardo de linguagem, que pode ser um sintoma comum nessa população, como alguns estudos já sugerem ${ }^{40}$.

Tal revisão aponta, portanto, que o trabalho pode iniciar precocemente, antes que o sintoma de linguagem se instale, por meio do trabalho interdisciplinar entre os profissionais de Fonoaudiologia e Psicologia já no acompanhamento do período puerperal e também nas consultas pediátricas de rotina que ocorrem durante os primeiros meses de vida.

Essa revisão indica o quão importante é a diferenciação do período baby blues ${ }^{14}$ da depressão no primeiro mês pós-parto e o acompanhamento da mãe e, não só do bebê, nos primeiros meses de vida ${ }^{32-34}$.

A partir da análise dos estudos apresentados nesta revisão, pode-se observar que a formação vincular da díade mãe-bebê nas interações precoces são fundamentais para o desenvolvimento infantil, especialmente para aquisição da linguagem. Por isso, é urgente que as avaliações fonoaudiológicas incluam as interações com as figuras parentais e não se restrinjam aos comportamentos infantis, como ocorrem em muitas escalas evolutivas da infância.

\section{CONCLUSÃO}

A revisão de literatura realizada demonstra que a depressão materna afeta não só a mãe, mas também o desenvolvimento global do bebê. Os estudos apontam que os efeitos da depressão materna podem resultar negativamente no desenvolvimento da criança, podem desencadear desordens lingüísticas, comportamentais, afetivas, cognitivas e sociais, bem como alterações da atividade cerebral. Contudo, a extensão do impacto da depressão materna para o bebê depende de vários fatores como idade da criança, temperamento infantil, a cronicidade do episódio depressivo materno e do próprio estilo interativo da mãe deprimida. Portanto, a depressão materna, em um determinado momento após o nascimento, pode interferir no estabelecimento do vínculo mãe-bebê e, por isso, deve ser tratada o mais precocemente possível. O fonoaudiólogo é um dos profissionais que pode estar fazendo parte da equipe que irá detectar e abordar esse tema em sua atuação nas equipes de saúde, nos distintos níveis de complexidade.

\begin{abstract}
Background: this study analyzed the scientific production of the last five years on maternal depression, particularly the repercussions of depression on mother-child interaction and its implications for child development. Purpose: to check the effects of maternal depression on mother-child interaction, and examine its implications on the child development, especially on language. Conclusion: the conducted literature review shows that maternal depression affects not only the mother but also the overall development of the baby. The studies showed that the effects of maternal depression may negatively affect child development, language can trigger disorders, behavioral, affective, cognitive and social. Therefore, maternal depression, at a certain time after birth, may come to interfere with bonding between mother and baby and therefore should be treated as early as possible.
\end{abstract}

KEYWORDS: Depression, Postpartum; Child Development 


\section{REFERÊNCIAS}

1. De Lemos, C.T.G. Los procesos metafóricos y metonímicos como mecanismos de cambio. Substratum I-1; 1992.p.121-35.

2. Aguado G. Dimensões perceptivas, sociais, funcionais e comunicativas do desenvolvimento da linguagem. In: Chevrie-Muller C, Narbona $\mathrm{J}$. A linguagem da criança: aspectos normais e patológicos. 2a ed. Porto Alegre: Artmed; 2005. p. 71-87.

3. Mogford K, Bishop D. Desenvolvimento da linguagem em condições normais. In: Bishop D, Mogford K, organizadores. Desenvolvimento da linguagem em circunstâncias excepcionais. Rio de Janeiro: Revinter; 2002. p.1-26.

4. Azevedo KR, Arrais AR. O mito da mãe exclusiva e seu impacto na depressão pós-parto. Psicol. Reflex. Crit. 2006; 19 (2): 269-76.

5. Recchia IC, Ramos SAP. Funções parentais e dialogia mãe-filho com dispraxia verbal. Revista Psicologia em Estudo, Campinas.No prelo.2010.

6.Frizzo GB, Piccinini CA. Interação mãe-bebê em contexto de depressão materna: aspectos teóricos e empíricos. Psicol Estud. 2005; 10:47-55.

7. Furst MCG, Schrroeder AT, Valle VH, Golbert $M B$, Escosteguy N.Depressão pós-parto e suas repercussões na interação precoce mãe-bebê.Acta méd. 2007; 28:496-506.

8. Surkan PJ, Kawachi I, Ryan LM, Berkman LF, Carvalho VLM, Peterson KE. Maternal depressive symptoms, parenting self-efficacy, and child growth. Am J Public Health. 2008; 98:125-32.

9. Motta M, Lucion A \& Manfro G. Efeitos da depressão materna no desenvolvimento neurobiológico e psicológico da criança. Revista de Psiquiatria. 2005; 27 (2): 165-76.

10. Murray L, Halligan SL, Adams G, Patterson $P$ \& Goodyer IM. Socio emotional development in adolescents at risk for depression. The role of maternal depression and attachment style. Development-and-Psychopathology. 2006; 18(2):489-516.

11. Grote $\mathrm{V}$ et al. Maternal postnatal depression and child growth: a European cohort study. BMC Pediatrics.2010; 10(14):1471-2431.

12. Santos JHPO, Silveira MFA, Gualda DMR. Depressão pós-parto: um problema latente. Rev Gaúcha Enferm.2009; 30(3): 516-24.

13. Felix GMA, Gomes APR, França PS.Depressão no ciclo gravídico-puerperal.Comun. ciênc. Saúde. 2008;19(1):51-60.

14. Saraiva ERA, Coutinho MPL. O sofrimento psíquico no puerpério: um estudo psicossociológico. Rev.Mal-Estar Subj.2008; 8(2):505-27.
15. Borsa JB, Dias ACG. Considerações Acerca da Relação Mãe - Bebê da Gestação ao Puerpério. Revista Contemporânea - Psicanálise e Transdisciplinariedade.2007; (2): 310-21.

16. Alt MB, Silvia PC. Maternidade e depressão: impacto na trajetória de desenvolvimento. Psicol. Estud. 2008; 13(2): 389-94.

17. Schwengber DDS, Piccinini CA. A experiência da maternidade no contexto da depressão materna no final do primeiro ano de vida do bebê. Estud Psicol. 2005; 20 (3): 351-60.

18. Perosa GB et al. Sintomas depressivos e ansiosos em mães de recém-nascidos com e sem malformações. Rev. Bras. Ginecol. Obstet. 2009;31(9): 433-9.

19. Adewuya AO, Ola BO, Aloba OO, Mapayi BM, Okeniyi JA. Impact of postnatal depression on infants' growth in Nigeria. J Affect Disord. 2008; 108:191-3.

20. Schmidt E, Piccoloto N, Muller M. Depressão pós-parto: fatores de risco e repercussões no desenvolvimento infantil. Psico USF. 2005; (10): 61-8.

21. Costa R, Pacheco A, Figueiredo B. Prevalência e preditores de sintomatologia depressiva após o parto. Rev. psiquiatr. clín. 2007; 34(4): 157-65.

22. Frizzo G, Piccinini C. Depressão materna e a interação triádica pai-mãe-bebê. Psicol Reflex Crít .2007; 20:351-60.

23. Goldbort J. Transcultural analysis of postpartum depression. MCN Am J Matern Child Nurs 2006; 31:121-6.

24. Lee LC, Halpern CT, Hertz PI, Martin SL, Suchindran CM. Child care and social support modify the association between maternal depressive symptoms and early childhood behaviour problems: a US national study.2006; 60(4): 305-10.

25. Akman I, Kuscu K, Ozdemir N, Yurdakul Z, Solakoglu M, Orhan L, Karabekiroglu A, Ozek E.Mothers' postpartum psychological adjustment and infantile colic. 2006; 91(5): 417-9.

26. National Institute of Child Health and Human Development (NICHD) Research Network. Chronicity of maternal depressive symptoms, maternal sensitivity, and child functioning at 36 months. Nichd Early Child Care Research Network. Dev Psychol. 1999;5(5):1297-310.

27. Evans $\mathrm{J}$ et al. Emotional symptoms in children: The effect of maternal depression, life events, and COMT genotype. 2009; 150(2): 209-18.

28. Wedel HE, Wall ML, Maftum MA. Sentimentos da mulher na transição gestação-puerpério.Nursing. 2008; 11(126):501-6. 
29. Smith JC, Cumming A, Xeros CS. A decade of parent and infant relationship support group therapy programs. Int J Group Psychother.2010; 60(1): 59-89.

30. Vliegen $\mathrm{N}$, Luyten $\mathrm{P}$, Besser $\mathrm{A}$, Casalin $\mathrm{S}$, Kempke S, Tang E. Stability and change in levels of depression and personality: a follow-up study of postpartum depressed mothers that were hospitalized in a mother-infant unit.J Nerv Ment Dis. 2010;198(1): 45-51.

31. Conroy S, Marks MN, Schacht R, Davies HA, Moran P. The impact of maternal depression and personality disorder on early infant care. Soc Psychiatry Epidemiol. 2010; 45(3): 285-92.

32. Field T. Postpartum depression effects on early interactions, parenting, and safety practices: a review.Infant Behav Dev. 2010; 33(1): 1-6.

33. Schwengber, D. D. S. \& Piccinini, C. A. Protocolo de análise da interação mãe-bebê de um ano de idade durante a interação livre. Trabalho não publicado, Universidade Federal do Rio Grande do Sul, Porto Alegre. 2001.

34. Wan MW, Green J. The impact of maternal psychopathology on child-mother attachment. Arch Womens Ment Health. 2009;12(3): 123-34.
35. Van DKT, Hosman CM, Riksen WJM, Hoefnagels C. Correlates of depressed mothers' sensitivity toward their infants: the role of maternal, child, and contextual characteristics. J Am Acad Child Adolesc Psychiatry. 2007; 46(6): 747-56.

36. Jung V, Short R, Letourneau N, Andrews D. Interventions with depressed mothers and their infants: modifying interactive behaviours. J Affect Disord. 2007; 98 (3): 199-205.

37. Cheng S, Kondo N, Aoki Y, Kitamura Y,Takeda $Y, Y a m a g a t a$. The effectiveness of early intervention and the factors related to child behavioural problems at age 2: a randomized controlled trial. Early Hum Dev. 2007; 83(10): 683-91.

38. Moehler $\mathrm{E}$ et al. Childhood behavioral inhibition and maternal symptoms of depression. Psychopathology. 2007; 40 (6): 446-52.

39. McLaren L, Kuh D, Hardy R, Mishra G. Postnatal depression and the original mother-child relationship: a prospective cohort study. J Affect Disord. 2007; 100(1-3): 211-9.

40. Crestani AH, Rosa F, Pretto J, Ramos SAP. A Experiência da Maternidade e a Dialogia Mãe- Filho com Distúrbio de Linguagem. Revista Cefac.No prelo. 2010.
http://dx.doi.org/10.1590/S1516-18462011005000085

RECEBIDO EM: 21/05/2010

ACEITO EM: 07/04/2011

Endereço para correspondência:

Janaína Pereira Pretto Carlesso

Rua General Neto, 675/ 401

Santa Maria - RS

CEP: 97050-241

E-mail: janapretto@yahoo.com.br 